\title{
Local Effects of ECRH on Argon Transport in L-Mode Discharges at ASDEX Upgrade
}

\author{
M Sertoli, C Angioni, R Dux, R Neu, T Pütterich, V \\ Igochine and the ASDEX Upgrade Team \\ Max-Planck-Institut für Plasmaphysik, EURATOM Association, Garching, \\ Germany \\ E-mail: marco.sertoli@ipp.mpg.de
}

\begin{abstract}
The transport of argon as trace impurity has been investigated in electron cyclotron resonance heated L-mode discharges at ASDEX Upgrade to test recent theories predicting the rise of an outward impurity convection. The profiles of the argon transport coefficients for $r / a<0.65$ have been determined by analysing the linear flux-gradient dependency of the total argon ion density evolution after the puff. A new methodology to experimentally obtain the total impurity ion density from the integrated use of two spectroscopic diagnostic and the 1D impurity transport code STRAHL has been developed. Results confirm the enhancement in diffusivity and the rise of the positive convection observed in previous studies, but show for the first time how these effects are strongly localized around the electron cyclotron resonance heating deposition radius. These experimental results are found in promising qualitative agreement with a set of quasi-linear gyrokinetic simulations with the code GS2.
\end{abstract}

PACS numbers: 52.25.Fi, 52.35.Ra, 52.50.Sw, 52.55.Fa, 52.65.Tt, 52.70.La, 52.70.$\mathrm{m}$

Submitted to: Plasma Phys. Control. Fusion

Keywords: tokamaks, X-rays, impurity transport, ECR heating, gyrokinetic simulations, neoclassical transport

\section{Introduction}

The use of tungsten (W) as a plasma-facing-component (PFC) in ASDEX Upgrade (AUG) has raised important issues on the control of central impurity accumulation. While light impurities are typically completely ionized in the core plasma (e.g. carbon), heavy impurities such as $\mathrm{W}$ are still only partially ionized. Moreover, in conditions where the anomalous transport is suppressed, heavier impurities tend to accumulate more than lighter ones [1], as described by neoclassical theory. The consequent strong central radiative losses due to line radiation then lead to a reduction of the fusion power. It is therefore of major interest for present and future devices to understand how to externally act on the anomalous transport of an impurity, enhancing it where needed so to prevent central accumulation and the consequent strong radiative losses to occur. 
The use of intense central electron heating could be a possible solution since it has been observed to lead to an increase in impurity diffusivity and to a suppression of the convective pinch, or even to the rise of an outward impurity convection $[2,3,4,5,6,7,8,9]$. The underlying physics mechanisms of these effects are on the other hand still not clear. Recent theoretical studies predict the rise of a positive (outward) convection due to density fluctuations caused by parallel compression [10]. The instability should be restricted to plasma regions with low safety factor $q$, low shear $s$ and $R / L_{T e}>2 \cdot R / L_{T i}$, where $L_{T}=-T /(d T / d r)$ and $R$ is the major radius. The first two conditions are typical of the centre of a tokamak plasma while the last one is not often encountered in the centre of H-mode plasmas, of major interest for commercial fusion devices. The operational space in which an outward convection may occur has thus to be investigated and further experimental evidence has to be produced to confute or confirm the theory.

A set of electron cyclotron (resonance) heated (ECRH) low-confinement mode (L-mode) experiments have been performed at AUG to study the dependence of the transport of argon (Ar) on the heating deposition profile. For a fixed total ECRH power delivered, deposition radii ranging from $r / a \sim 0.2$ to 0.65 have been tested. Trace argon puffs have been performed during the flat-top phases of the discharges to evaluate the profiles of the argon transport coefficients for $r / a<0.6$. These have been determined by analysing the linear flux-gradient dependence of the total Ar ion density evolution after the impurity injection. A new methodology for the experimental determination of the total impurity ion density has been developed.

The paper is divided in four parts. Section 2 reviews the flux-gradient relation for the determination of the impurity transport coefficients. Section 3 gives a detailed explanation of the new experimental method for the determination of the total impurity ion density. The results for L-mode discharges are described in section 4 . The method is benchmarked against $\chi^{2}$-minimization methods and compared with neoclassical and turbulence theoretical estimates. Conclusions and an outlook are given in section 5 .

\section{Determination of the Impurity Transport Coefficients}

The radial transport of an impurity $A$ of atomic number $Z$ can be described through $Z+1$ coupled differential equations. Each equation describes the flux $\Gamma_{A, q}(r, t)$ and density $n_{A, q}(r, t)$ evolution of a different ionization stage $q$, from the neutral to the fully stripped ionization stage. By choosing a radial coordinate such as $r=\sqrt{V /\left(2 \pi^{2} R_{\text {axis }}\right)}$ where $V$ is the volume within the flux surface and $R_{a x i s}$ the major radius of the plasma axis and averaging all quantities over the flux surface, each equation can be written in cylindrical geometry while still accounting for non-circular plasma cross sections [11]:

$$
\frac{\partial n_{A, q}(r, t)}{\partial t}=-\frac{1}{r} \frac{\partial}{\partial r}\left[r \Gamma_{A, q}(r, t)\right]+Q_{A, q}(r, t)
$$

The term $Q_{A, q}(r, t)$ describes the external sources and sinks, and connects the various ionization stages through ionization and recombination rates. By summing these equations over all the ionization stages except the neutral, the source term simplifies to $Q_{A, 1}=n_{e} S_{A, 0} n_{A, 0}-\left(n_{e} \alpha_{A, 1}+n_{H} \alpha_{A, 1}^{c x}\right) n_{A, 1}$. Since the ionization to the $1^{\text {st }}$ stage $S_{A, 0}$, as well as recombination $\alpha_{A, 1}$ and charge exchange $\alpha_{A, 1}^{c x}$ to the neutral atom take place outside or just inside the separatrix, for core transport analysis $Q_{A, 1}=0$. 
Equation (1), which is now an equation for the total impurity ion density, can then be inverted to evaluate the total ion flux:

$$
\Gamma_{A}(r, t)=-\frac{1}{r} \int_{0}^{r} \frac{\partial n_{A}\left(r^{\prime}, t\right)}{\partial t} r^{\prime} d r^{\prime}
$$

Assuming a diffusive and convective ansatz for this flux, the transport coefficients can be recovered from the slope (D) and offset (v) of the linear fit of the normalized flux on the normalized density gradient:

$$
\frac{\Gamma_{A}(r, t)}{n_{A}(r, t)}=-\frac{D(r)}{n_{A}(r, t)} \frac{\partial n_{A}(r, t)}{\partial r}+v(r)
$$

where $D$ and $v$ are assumed equal for all ionization stages and constant in time. The latter assumption is valid only if the injected impurity is in "trace" concentration, affecting neither the main plasma parameters nor the transport characteristics (see e.g. $[12,13])$. The plasma geometry used for the evaluation of the flux surface averaged quantities in equation (1) is also assumed not to vary in time. Equation (3) will be referred to as " $G-F$ relation" from now on.

This formula is seldom used for the evaluation of the transport coefficients because of the difficulty in measuring the total impurity ion density experimentally. Previous applications to impurity transport [14] used charge exchange recombination spectroscopy (CXRS) while in a recent work [15] the method has been applied to study electron transport in-between sawtooth crashes within the $q=1$ surface. Other impurity transport studies (e.g. $[3,6,7,8,9]$ ) use instead predictive approaches adjusting the $D$ and $v$ profiles iteratively so to reproduced the measured spectroscopic data and minimize the $\chi^{2}$ of the simulated signals to the experimental ones (therefore called $\chi^{2}$-minimization methods).

The new solution for the application of the G-F relation proposed here makes an integrated use of the soft X-ray (SXR) diode camera diagnostic [16] and the 1D radial

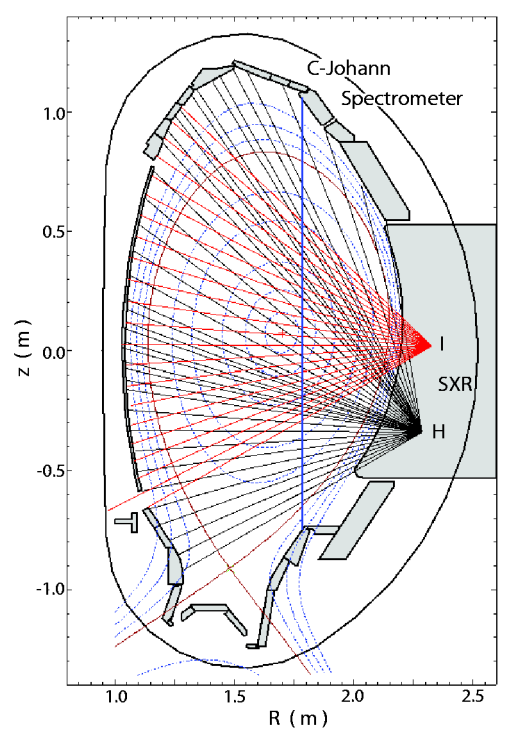

Figure 1. Lines of sight of the SXR cameras I and H (red and black) and of the C-Johann spectrometer (blue) on an ASDEX Upgrade cross-section. Plasma geometry as in the discharges analysed in section 5 . 
impurity transport code STRAHL $[17,11]$ for the determination of the total impurity ion density. The SXR diode diagnostic provides a fast $(<2 \mathrm{MHz})$ line of sight (LoS) integrated measurement of all the light emitted in a large spectral range $(\sim[1 ., 10.] \mathrm{keV}$ for the cameras considered here). The availability of a large number of LoS (in red and black in figure 1) allows precise 1D Abel inversion leading to a spatial resolution of $\sim 1 \mathrm{~cm}$ in the centre. With the use of the STRAHL code, this local emissivity profile can be transformed into an impurity density profile which can be then used in the G-F relation. Since equation (3) involves only relative values of the impurity density profiles, the absence of a trustworthy absolute calibration the SXR diodes does not affect the final result. On the other hand, the evaluation of absolute impurity ion density profiles is anyway of major interest. Specifically for argon, a re-scaling of the density profile evaluated through the SXR diagnostic can be performed through the use of the, the compact soft X-ray (CSXR) spectrometer [18, 19]. In order to avoid confusion with the well-established charge exchange recombination spectroscopy acronym $C X R S$, the CSXR spectrometer will be referred to as "compact Johann spectrometer", or C-Johann. As will be explained in section 3.1, despite its single LoS (in blue in figure 1) its absolute concentration and ion temperature measurements can be given an approximate radial localization. The total $n_{A r}$ profile evaluated from SXR can be therefore re-scaled to absolute values and, with the help of the neutral particle analyser (NPA) [20], approximate ion temperature profiles necessary for turbulent simulations can be delivered without the use of neutral beam injection (NBI).

\section{Evaluation of the total impurity ion density}

The emissivity of an impurity $A$ in the SXR region can be modelled as:

$$
\epsilon_{s x r}(r, t)=n_{e}(r) n_{A}(r, t) \sum_{q} f_{A, q}(r, t) k_{A, q}^{s x r}(r)
$$

where $n_{e}$ is the electron density, $n_{A}$ the total impurity density, and the summation is performed over all the ionization stages $q$. The photon emissivity rate coefficients $k_{A, q}^{s x r}(r)$, expressed in units of photons $\cdot m^{3} \cdot s^{-1}$, consider all the radiative transitions of ionization stage $q$ in the sensitivity range of the diagnostic. They depend solely on electron temperature and density and are extracted from the ADAS database [21]. The time evolution and equilibrium values of $n_{A}(r, t)$ depend only on the transport characteristics of the impurity while the fractional abundances $f_{A, q}(r, t)=n_{A, q} / n_{A}$ exhibit a strong dependence on the electron temperature and a rather weak dependence on transport variations.

The dependence of $f_{A, q}$ on electron temperature is directly correlated to ionization and recombination rates, while charge exchange with background neutrals can be neglected. This is demonstrated by figure $2 \mathrm{c}$ which shows the equilibrium $f_{A, q}(r)$ of the last four ionizations stages of argon calculated in coronal-approximation neglecting and including charge exchange reactions (full and dashed black lines respectively). Main plasma parameters as shown in figures $2 \mathrm{a}$ and $2 \mathrm{~b}$ have been used, the background neutral density estimated by evaluating the ionization equilibrium of deuterium and multiplying the electron density profile by the fractional abundance of neutral deuterium. This profile has been then multiplied by a factor 10 so to better visualize the differences in ionization equilibrium in figure $2 \mathrm{c}$ when chargeexchange reactions are included. Since the assumption of coronal equilibrium is quite a crude approximation, considering a typical set of transport coefficients observed in 

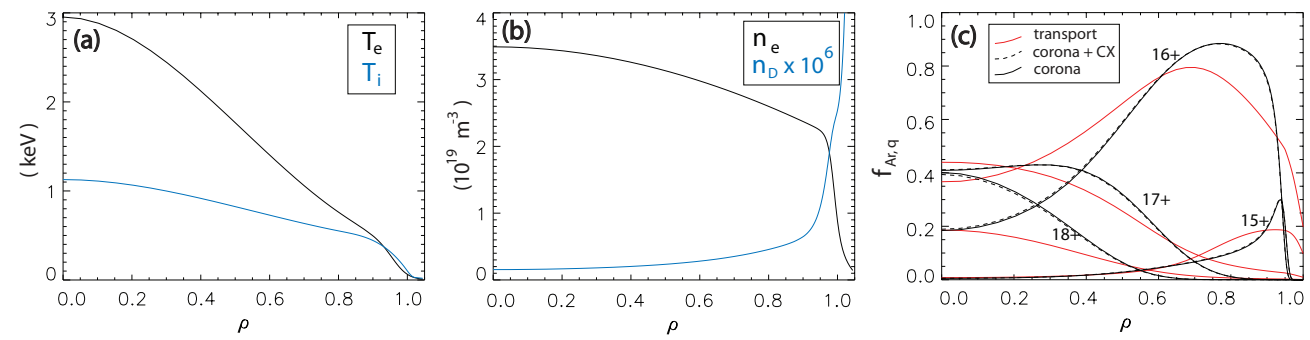

Figure 2. Sensitivity of the argon fractional abundance to charge exchange reactions and transport (c) with fixed main plasma parameters (a) and (b) $\left(n_{D}\right.$ standing for background neutral deuterium).

ASDEX Upgrade (shown in figure 3a and 3b in red), the ionization equilibrium changes substantially (red in figure 2c). This deviation can be understood by noting that the ionization equilibrium time scales are smaller than those of the transport processes involved, but approximately of the same order of magnitude. Specifically for the discharges analysed in the next sections, the influx phase after an argon injection lasts typically $150-200 \mathrm{~ms}$ (bottom plots in figure 8). Evaluating the time-dependent ionization equilibrium in coronal approximation, the equilibration time is typically of few tens of $m s$. In order to correctly model the fractional abundances it will therefore be necessary to perform an a priori assumption on the transport coefficients.

Choosing therefore the set of "typical" transport coefficients, an impurity injection can be simulated with STRAHL. The coupled differential equations (1) are solved iteratively using equilibrium reconstruction, electron density and temperature profiles from the experiment. By taking the filtered emissivity coefficients $k_{A, q}^{s x r}(r)$ from the ADAS database, the full SXR emissivity profile evolution due to the impurity injection can be simulated. Dividing this emissivity profile at equilibrium $\epsilon_{s x r}\left(r, t_{e q}\right)$ by the simulated total impurity ion density profile at the same time-point $n_{A}\left(r, t_{e q}\right)$, the resulting normalized emissivity function $\epsilon^{\prime}(r)(5)$ will depend only on the fractional abundance and the main plasma parameters. Since the latter are constant during the time-range of interest, this function depends only on the chosen transport parameters. As will be demonstrated shortly, due to both the small sensitivity of $f_{A r, q}$ relatively to large variations in $D$ and $v$ with respect to the "typical" set and to a balancing of the emissivity profiles of the various ionization stages in the SXR region, large variations in transport do not affect this function dramatically.

$$
\epsilon^{\prime}(r)=\frac{\epsilon_{s x r}\left(r, t_{e q}\right)}{n_{A}\left(r, t_{e q}\right)}=n_{e}(r) \sum_{q} f_{A, q}\left(r, t_{e q}\right) k_{A, q}^{s x r}(r)
$$

The time-point $t_{e q}$ coincides with the moment when the impurity influx has ended and the impurity ion density in the centre is maximum, but in principle any time point after the impurity has reached the centre could be used, as long as $n_{A}(r, t) \neq 0$ up to the centre of the plasma.

The low sensitivity of $\epsilon^{\prime}(r)$ to variations in transport with respect to the a priori assumption can be rigorously demonstrated by performing STRAHL simulations with different $D$ and $v$ profiles. Using the electron profiles shown in figure $2 \mathrm{a}$ and $2 \mathrm{~b}$, the diffusion coefficient profile has been scanned over one order of magnitude (figure 3a), the convection velocity set imposing $\frac{v}{D}=\frac{1}{n_{e}} \frac{d n_{e}}{d r}$, ranging from approximately zero to strongly negative (figure $3 \mathrm{~b}$ ). The effect of the strong variation in $D$ and $v$ is clearly 

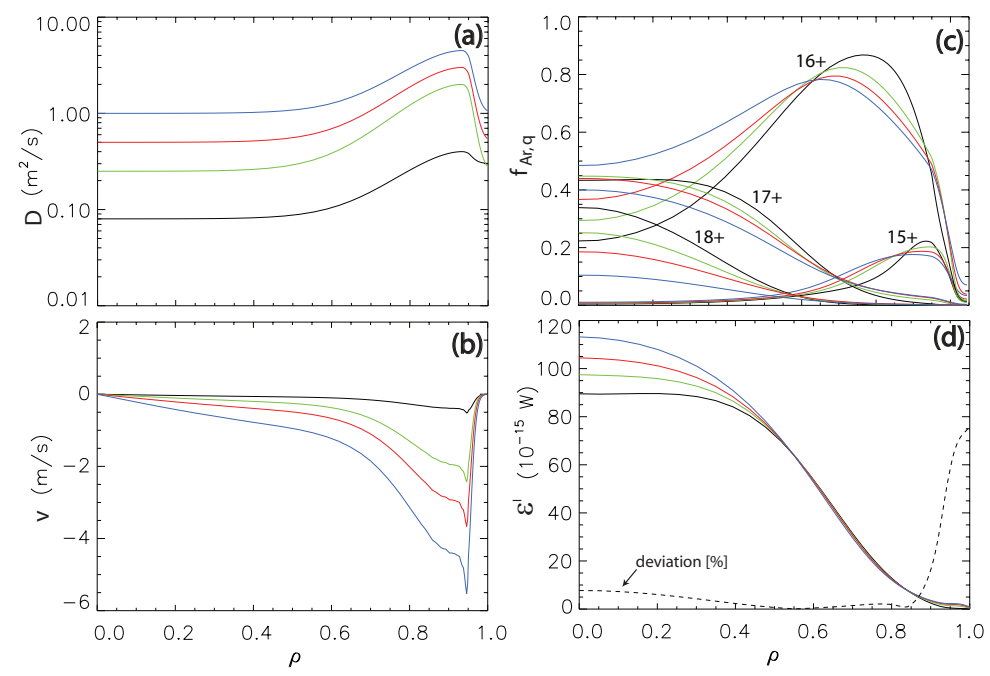

Figure 3. Dependence of $\epsilon^{\prime}$ on transport parameter variations: scan in $D$ (a) and $v$ profiles (b), fractional abundance of the last four Ar ionization stages (c), $\epsilon^{\prime}$ for the different cases (d) (dashed line is the percentage deviation from the mean value).

visible in the profiles of the fractional abundances (3c) but, after having included the contributions of the emissivity rate coefficients $k_{A, q}^{s x r}(r)$, the $\epsilon^{\prime}(r)$ function (3d) shows a maximum $10 \%$ deviation (dashed line) with respect to the mean value. The strong deviation for normalized poloidal flux radius $\rho=\rho_{\text {pol }}>0.8$ is due to a drop in sensitivity of the considered SXR diodes at approximately $1 \mathrm{keV}$ photon energy. Since most of the detected light originates from transitions which are excited by electron collisions, the radius where $T_{e}>1 \mathrm{keV}$ has been chosen as safety limit for the analysis.

After demonstrating the low impact of transport on $\epsilon^{\prime}(r)$, this function can be used to evaluate the total experimental impurity ion density from the experimental SXR data. The experimental impurity emissivity $\epsilon_{s x r}^{e x p}(r, t)$ is recovered by performing a 1D Abel inversion of the LoS-integrated SXR signals after having subtracted the background contributions prior to the impurity puff. Dividing this profile at each time point by $\epsilon^{\prime}(r)$, the experimental total impurity ion density profile evolution in time can be evaluated.

$$
n_{A}^{s x r}(r, t)=\frac{\epsilon_{s x r}^{e x p}(r, t)}{\epsilon^{\prime}(r, t)}=\frac{\epsilon_{s x r}^{e x p}(r, t)}{n_{e}(r) \sum_{q} f_{A, q}\left(r, t_{e q}\right) k_{A, q}^{s x r}(r)}
$$

Because of the absence of a trustworthy absolute calibration of the diodes, only the relative values of $n_{A}^{s x r}(r, t)$ can be trusted. As will be explained in detail in the next section, if an absolutely calibrated spectroscopic LoS-integrated measurement is available, the profile can be re-scaled to absolute values. This is restricted to measurements on sufficiently high ionization stages whose emissivity has a peak within the sensitivity limit of the SXR diagnostic. For argon in particular, this re-scaling can be performed through the measurements from the C-Johann spectrometer on He-like $(16+)$ resonance lines. 


\subsection{Re-scaling of the total argon density}

The CSXR (or C-Johann) spectrometer is a crystal spectrometer of the Johann type which makes use of a large $\left(20 \times 35 \mathrm{~mm}^{2}\right)$ quartz crystal $(10 \overline{1} 0)$ asymmetrically cut at an angle $21.9^{\circ}$ and spherically bent to a radius of $R=500 \mathrm{~mm}$. The system has been optimised for measurements of He-like Ar resonance lines at $\sim 0.395-0.399 \mathrm{~nm}$, corresponding to Bragg angles of $\theta_{B} \sim 68.3^{\circ}-70^{\circ}$. The incident rays are quasinormal to the crystal surface, strongly reducing the typical spherical aberration of such crystals and leading to an experimental spectral resolution $\lambda / \Delta \lambda \sim 4000$. This translates in an instrument function corresponding to a minimum detectable argon temperature of $430 \mathrm{eV}$. The instrument has been absolutely calibrated in intensity through the use of a custom X-ray tube that simulates the extended plasma source and can therefore deliver an absolute measurement of the argon concentration averaged over its single LoS. The high luminosity of the instrument guarantees high signal to noise ratio (SNR) for argon concentrations as low as $\sim 10^{-5}$ (depending on plasma parameters). LoS-integrated measurements of the He-like argon ion temperature, rotation velocity and concentration are routinely performed at $5 \mathrm{~ms}$ repetition time on ASDEX Upgrade. More in-depth information on the spectrometer characteristics and absolute calibration can be found in [18].

Despite the integration over a single LoS, an approximate radial localization can be given to these measurements by modelling the He-like Ar emissivity characteristics. The detected intensity of one of the resonance transitions $\lambda$ of the He-like Ar ion is the integral over the LoS of the local emissivity profile:

$$
\epsilon_{\lambda}(r, t)=n_{e}^{2}(r, t) \cdot c_{A r}(r, t) \cdot f_{H e-l i k e} A r(r, t) \cdot P E C_{\lambda}(r, t)
$$

The terms in this equation are the same as those in (4), but only the fractional abundance of the emitting ion (He-like Ar) has to be considered, and the emissivity rate $\left(P E C_{\lambda}\right)$ refers to the single observed transition $\lambda$. Furthermore, the total impurity (argon) density has been substituted with it's concentration $c_{A r}=n_{A r} / n_{e}$, thus leading to the quadratic dependence on electron density. By modelling equation (7) for each time-point of the discharge, the first moment of the distribution $\epsilon_{\lambda}(r, t)$ with respect to $\rho$ will give an estimate of the mean position of emissivity while the second moment, the width of the distribution, can be interpreted as the error on the mean (left and right respectively in equation (8)).

$$
\bar{\rho}=\frac{\int \rho \epsilon_{\lambda} d l}{\int \epsilon_{\lambda} d l} \quad \sigma_{\rho}=\sqrt{\frac{\int(\rho-\bar{\rho})^{2} \epsilon_{\lambda} d l}{\int \epsilon_{\lambda} d l}}
$$

Since this procedure is performed for each time-point in the discharge (as in figure 4d) the fractional abundance is evaluated in coronal approximation accounting for the experimental $n_{e}$ and $T_{e}$ profiles only, thus speeding up the computation. The sensitivity of $\bar{\rho}$ to transport variations has been tested with the same STRAHL simulations used for $\epsilon^{\prime}$ (figure 3), resulting in a maximum uncertainty of $\sim 1 \mathrm{~cm}$ (approximately equal to $<0.05$ in $\rho$ within mid radius), much smaller than the $\sigma_{\rho}$ [18].

A consistency check of the values obtained for $\bar{\rho}(t)$ has been performed by comparing the argon temperatures $T_{A r}(t)$ from the C-Johann spectrometer to the profile-resolved carbon temperatures $T_{C}(r, t)$ from charge exchange. The profile of the carbon temperatures are linearly interpolated on $\bar{\rho}(t)$ and over-plotted on the argon temperatures from the C-Johann spectrometer in figure $4 \mathrm{~b}$. The resulting 

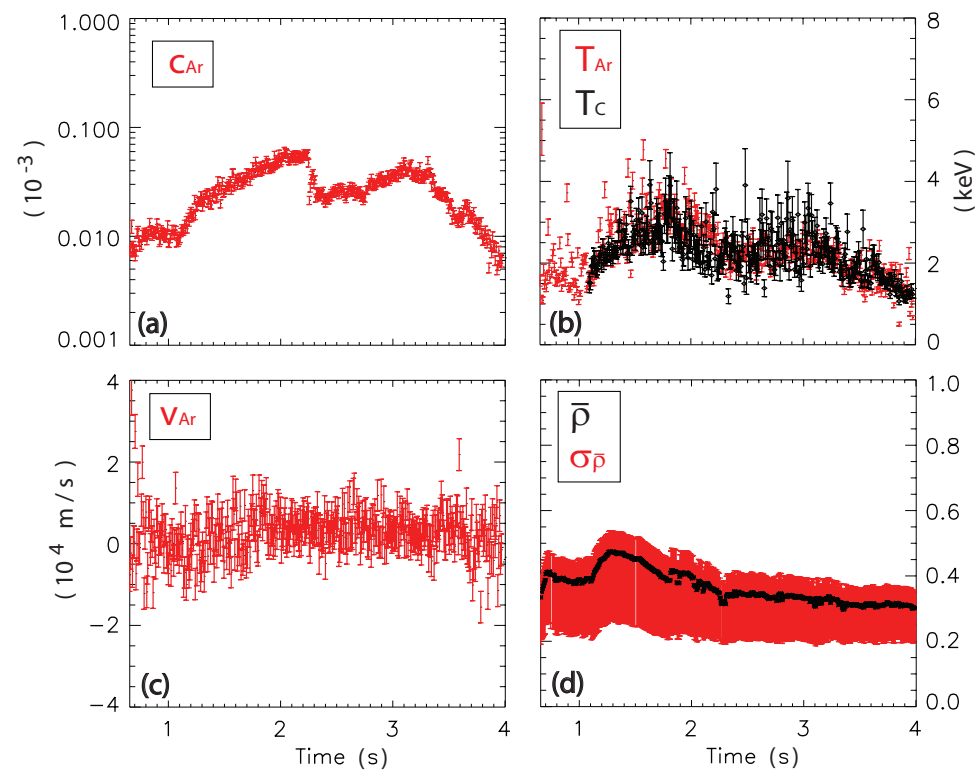

Figure 4. C-Johann spectrometer measurements for discharge \#23378. $c_{A r}(t)$ (a); $T_{A r}(t)$ in red and $T_{C}(t, \bar{\rho})$ from charge-exchange in black (b); $v_{A r}(t)$ (c); $\bar{\rho}(t)$ in black, $\sigma_{\rho}(t)$ in red (d).

time evolution of $T_{C}(t)$ (black) agrees very well with the $T_{A r}$ (red), thus confirming the quality of the evaluation of $\bar{\rho}(t)$. This radial localization applies to all the measurements from this diagnostic, thus also to concentration and rotation velocity measurements (figures $4 \mathrm{a}$ and $4 \mathrm{c}$ respectively).

Having validated the radial localization for the C-Johann measurements, a rescaling factor for the total argon density profile in (6) can be evaluated. This factor $f_{\text {res }}$ is defined as the ratio between the argon density from C-Johann spectrometer $c_{A r}\left(t_{e q}\right) \cdot n_{e}(\bar{\rho})$ at the equilibration time $t_{e q}$ and the value from SXR data at the same radial location and time point:

$$
\begin{aligned}
n_{A r}(r, t) & =n_{A r}^{s x r}(r, t) \cdot f_{\text {res }} \\
& =n_{A r}^{s x r}(r, t) \cdot \frac{c_{A r}\left(t_{e q}\right) \cdot n_{e}(\bar{\rho})}{n_{A r}^{s x r}\left(\bar{\rho}, t_{e q}\right)}
\end{aligned}
$$

Typical values for the correction factor are $f_{\text {res }} \sim 0.1$.

\subsection{SXR raw data treatment}

The rare use of the $G-F$ relation is also due to its high sensitivity to noise. If the SXR signals are noisy or affected by high frequency MHD activity, this will be reflected in the normalized fluxes $\Gamma / n$ and normalized gradients $(d n / d r) / n$, adding systematic errors to the linear fit. These effects can be suppressed by performing a pre-deconvolution $1 \mathrm{kHz}$ down-sampling of the raw SXR data, but this results also in a decrease of the number of points available for the analysis. 
The solution proposed here is to fit the SXR raw data with a model function before performing the Abel-inversion. By doing so, the time resolution of the raw data will be preserved, but the noise and fast MHD will be suppressed. The model function used is the so-called "modified hyperbolic tangent" function (MTANH), already employed for the fitting of electron temperature and density profiles in tokamak plasmas [22]. For the present application, the background evolution before the impurity puff (at 2.3 $\mathrm{s}$ in both plots in figure 5 ) is modelled through a $0^{\text {th }}$ order polynomial, the first part of the decay after the equilibration time through a $3^{r d}$ order one. The fitting procedure successfully filters the high frequency noise (example in figure 5a) and retains the gradients if the time evolution is fast (example in figure $5 \mathrm{~b}$, zoom in dashed-line oval). A simple $15 \mathrm{~ms}$ smoothing shown in red would instead retain some noise (5a) or flatten the gradients $(5 \mathrm{~b})$.
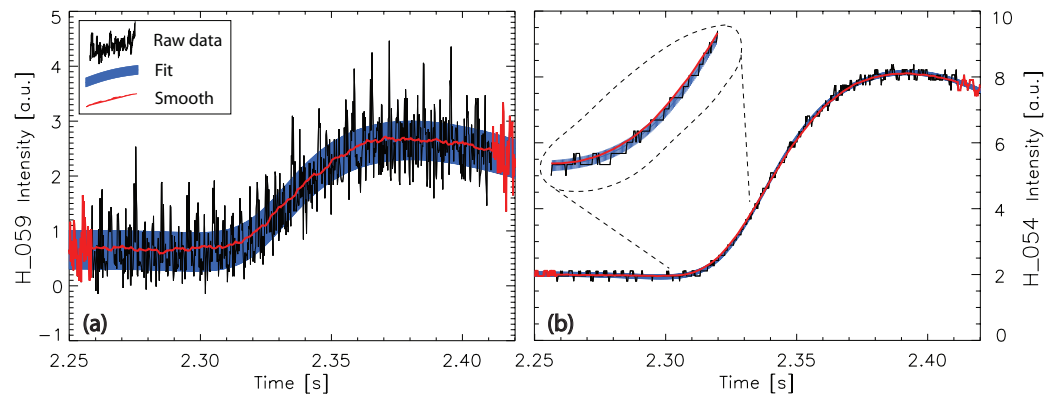

Figure 5. Example ot the fitting of SXR camera H, channels 54 (a) and 59 (b). Fit (blue), thickness of the line determined by the standard deviation of the fit to the raw-signal; raw data (black); 15 ms smoothing for comparison (red).

If strong low frequency sawtoothing activity is present, the raw data has to be treated differently. For relatively constant sawtooth frequency and an injection in "trace" concentration, the background sawtooth cycle will be constant in time. Similarly to what previously performed in [23], taking one of the last cycles before the impurity injection (at $2.1 \mathrm{~s}$ for both cases in figure 6) and subtracting its signature from all other cycles, the resulting signal will contain only contributions from the

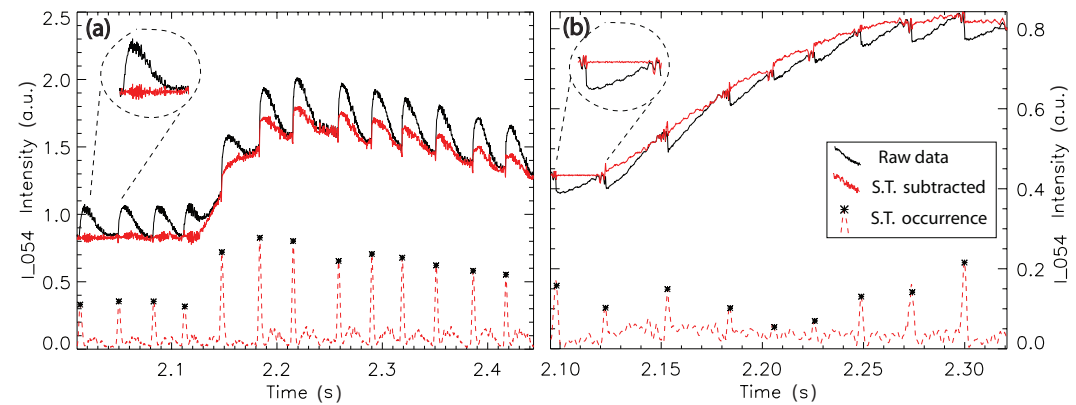

Figure 6. Background sawtooth subtraction of channel 54 of camera I for discharges \# 24709 (a) and \# 24916 (b). Raw signal (black), sawtooth subtracted (red), derivative of the raw signal (red dashed line) used for automatic localization of the sawtooth crashes (black dots). Reference background sawtooth cycle is shown in the dashed-line circles. 
injected impurity (red). As for the non-sawtoothing case, the total impurity density is then recovered applying (6) and (9) and the transport parameters evaluated through the $G-F$ relation.

\subsection{Summary on the method}

In order to give a comprehensive and reproducible path to evaluate the transport coefficients by means of the method explained in this section, an outline of the main steps which are executed is given here.

(i) Simulate the impurity injection with STRAHL using the experimental background plasma parameters and a set of "typical" transport coefficients (in red in figure 3).

(ii) Divide the simulated SXR emissivity profile by the simulated total impurity ion density profile at the same time point to extract the $\epsilon^{\prime}$ function (5).

(iii) Subtract the contributions of the background sawtooth cycles from the experimental SXR LoS-integrated signals and/or fit them to filter the noise and the fast MHD. Perform a 1D Abel inversion and subtract the background contribution prior to the impurity injection. Finally, divide each time point of this profile by $\epsilon^{\prime}$ to obtain the total argon density profile evolution (6). If available, re-scale this result through the impurity concentration measurement from an absolutely calibrated spectrometer (9).

(iv) For the time range of impurity influx and a chosen number of radial points, calculate the normalized density gradient and the normalized flux, and execute the linear fit to extract the diffusion and convection coefficients.

Repeating steps $(i)$ to $(i v)$ with the newly evaluated set of transport coefficients, the solution will converge to the best solution within typically a few cycles.

An example of the application of step $(i v)$ is shown in figure 7 for discharge \#24916. Since the argon contribution to the sawtooth cycle was negligible, fitting of the SXR raw data signal has also been performed after the background sawtooth subtraction (shown in figure 6), resulting in such smooth curves.
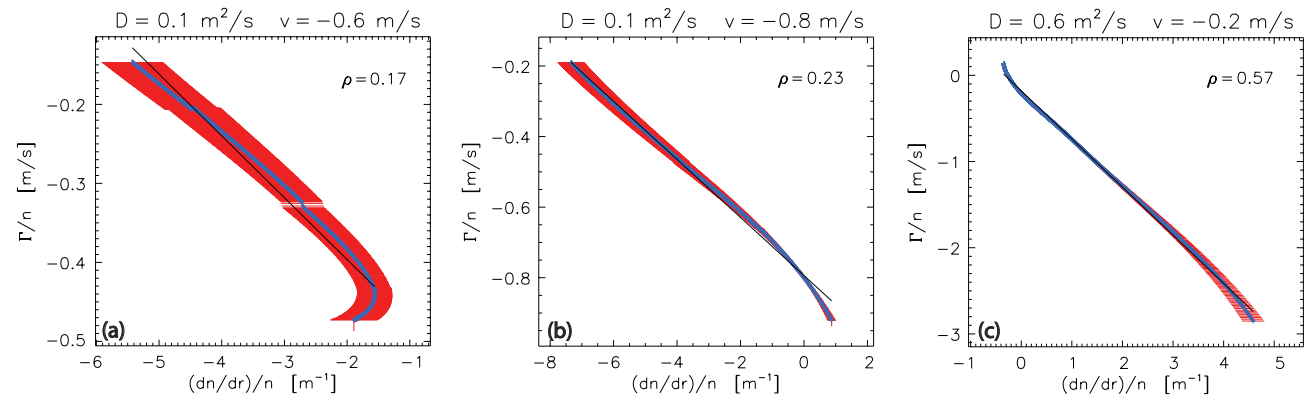

Figure 7. Gradient-Flux relation for discharge 24916 at different radial positions (points in blue, error bars in red, linear fit in black). $\rho$ of analysis is labelled on the top right of each plot, resulting $D$ and $v$ values on the plot title. 


\section{Experimental Results}

ECR-heated L-mode discharges have been performed to study the effects of ECRH deposition position on the transport of argon. The three discharges presented here have all been executed at $I_{p} \sim 800 k A, B_{t} \sim 2.3-2.4 T$ and plasma geometry as in figure 1. Main characteristics of the discharges are summarized in table 1 and in figure 8. The total ECRH power has been chosen so to keep the discharges just below the L-H transition thus avoiding the occurrence of ELMs and/or strong MHD which would further complicate the analysis procedure. The power was deposited in full at at one location for discharges \# 24916 and \# 24709, while it was distributed on two different locations for discharge \# 24648. The launching system was configured for heating only, driving a current $<1 \%$ of the plasma current. The sawtooth period was approximately constant, the sawtooth inversion radii changing slightly between discharges. The deposition radii and current drive have been calculated using the code TORBEAM [24], sawtooth inversion radii through the combined use of ECE and SXR diagnostics.

The main plasma parameters of all three discharges are stable throughout the

\begin{tabular}{|l|c|c|l|c|c|c|}
\hline Discharge \# & $\begin{array}{c}\bar{n}_{e} \\
\left(m^{-3}\right)\end{array}$ & $\begin{array}{c}P_{E C R H} \\
(M W)\end{array}$ & $\rho_{E C R H}$, (fraction) & $\rho_{\text {inv }}$ & $\begin{array}{c}\tau_{S T} \\
(m s)\end{array}$ & $q_{95}$ \\
\hline 24916 & 3.2 & 1.2 & 0.67 & 0.3 & 22 & 4.66 \\
24648 & 3.8 & 1.2 & $0.4(2 / 3) / 0.84(1 / 3)$ & 0.42 & 21 & 4.55 \\
24709 & 3.12 & 1.2 & 0.23 & 0.4 & 33 & 4.75 \\
\hline
\end{tabular}

Table 1. Central line averaged electron density $\bar{n}_{e}, P_{E C R H}$, deposition position $\rho_{E C R H}$ and power fraction if power is deposited at different radii (in brackets), sawtooth inversion radius $\rho_{i n v}$ and period $\tau_{S T}, q_{95}$ of the analysed discharges.
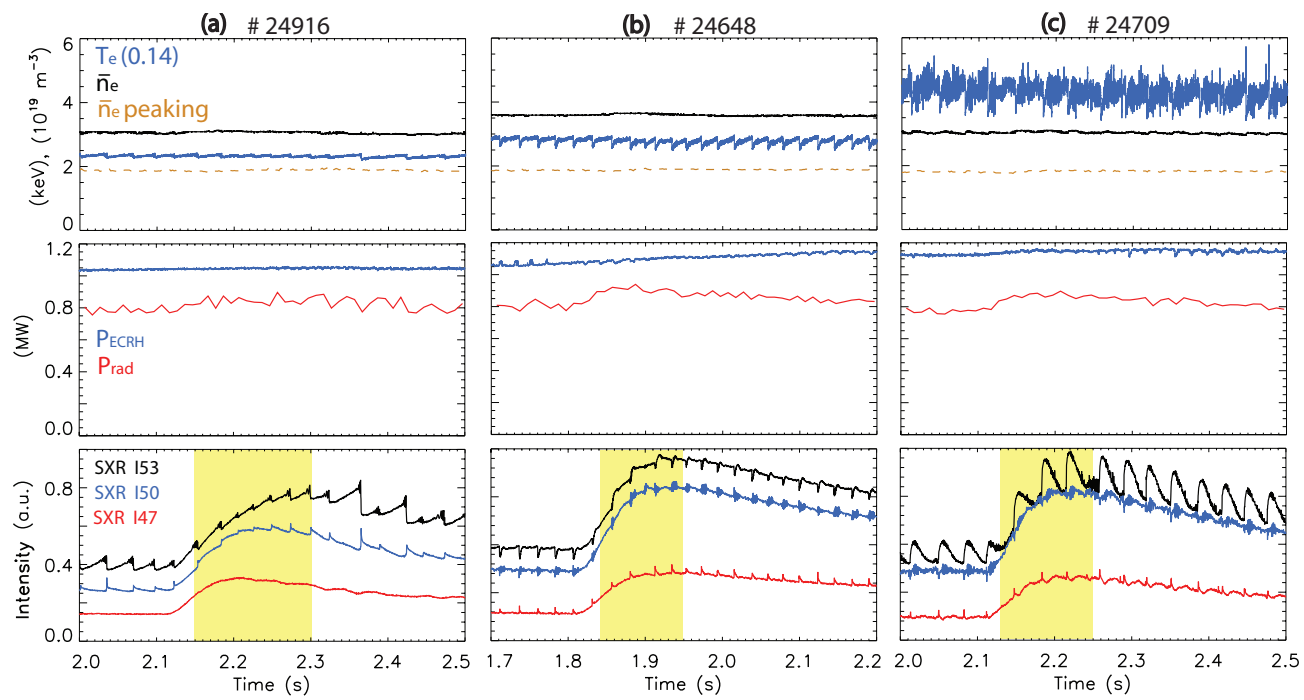

Figure 8. Top: central line-averaged electron density $\bar{n}_{e}$ (central interferometer channel), its peaking (centre and edge interferometer signal ratio), $T_{e}$ at $\rho \sim 0.14$ (from ECE). Centre: $P_{E C R H}$ and total radiated power $P_{\text {rad }}$. Bottom: signals from three LoS of the SXR diagnostic for the analysed discharges. 
time range of interest (top plots in figure 8). Due to the low amount of argon injected (from $0.015 \%$ to $0.07 \%$ ), a maximum $15 \%$ increase of the total radiated power is observed shortly after the argon injection (at $2.1 s$ for \#24916 and \#24709, at $1.8 s$ for \#24648). This leads to variations in $T_{e}$ and $n_{e}$ within few percent. The contribution of the injected impurity to the quasi-neutrality condition can be thus neglected and the impurity can be considered in the "trace" approximation required for the impurity transport analysis (other experimental impurity transport studies [13] had set a limit in $T_{e}$ and $n_{e}$ variations to $10 \%$, well above those observed in these discharges).

The change in ECRH deposition position is well reflected in the change in electron temperature and in that of the sawtooth cycle type (bottom plots). The latter evolves from so-called "standard" crashes (8a), through "humpback" (8b), to inverted "saturated" sawteeth for the most centrally heated discharge (8c). This discharge also exhibits strong $T_{e}$ oscillations in between sawtooth crashes caused by a strong $m / n=1 / 1$ mode coupled to other smaller modes. The definitions for the sawtooth crashes are taken from [25].

Since Ar is a recycling impurity and no information can be extracted from the decay of the impurity density, only the influx phase has been analysed (highlighted in yellow in the bottom plots in figure 8). Background plasma profiles have been thus averaged in this time-range and are shown in figure 9. A slight flattening of the electron density profile (9a, its normalized gradient length shown in 9d) is observed for the most centrally heated discharge (blue). The effect of the change in ECRH deposition position on the normalized electron temperature gradient length (9e) is very pronounced in the range $\rho=0.3-0.6$, increasing from $\sim 5$ to $\sim 8$ when passing from pure off-axis heating (red) to deposition radii close to or inside the $q=1$ surface (orange and blue respectively). Electron profiles are taken from integrated data analysis (IDA) fits [26, 27] of electron cyclotron emission (ECE) data for $T_{e}$ (points in figure $9 \mathrm{~b}$ ), Li-beam and interferometry data for $n_{e}$ (not shown). An exception to this

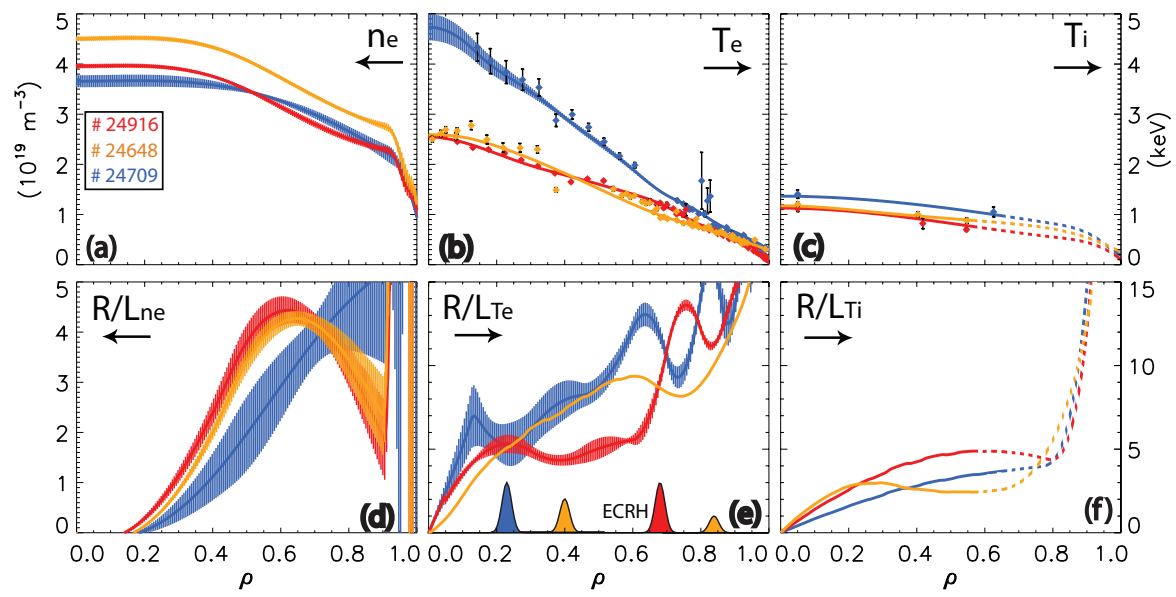

Figure 9. Electron density (a) and temperature profiles (b) (data points from ECE), ion temperature profile (c) (data points from the C-Johann and the NPA). Their normalized gradient lengths plotted respectively in (d-f). Error bars of mean profiles and of the ECE measurements are the standard deviation of the fits in the time range (absolute ECE errors $\sim 7 \%$ ). $T_{i}$ data-points given with absolute error bars. Gaussians in (e) represent the ECRH deposition radii. 
is discharge \#24648 whose $T_{e}$ fit was performed using a spline fit with tension. The reason for this is a large gap in the electron temperature data in the range $\rho \sim 0.3-0.5$ which lead to a strong drop in IDA $T_{e}$ fit at the ECRH deposition radius $\rho \sim 0.4$. The coloured bands represent the standard deviation of the IDA fits and of their normalized gradients within the time range. The width of this band for discharge \# 24709 is a direct consequence of the strong MHD activity.

Because of the absence of NBI injection, the $T_{i}$ profiles (figure $9 \mathrm{c}$ ) have been inferred from the three measurement points available from the C-Johann spectrometer and the NPA diagnostic, shown in figure as points with absolute error bars. Since the NPA diagnostic did not acquire during discharge \#24648, the values from the previous discharges were used (only difference being that the full ECRH power was deposited at $\rho \sim 0.4$ ). The resulting $T_{i}$ profiles were produced using the MTANH function with a $3^{r d}$ order polynomial fit for the core profile. This quite crude approximation well reproduces the available data-points (dashed lines for $\rho>0.6$ indicating the region where no data is available. ). These fits lead to estimates of $T_{e} / T_{i} \sim 3$ in the centre for discharge \#24709 (blue) and $R / L_{T e} \geq 2 \cdot R / L_{T i}$ around the ECRH deposition position for both \# 24709 and 24648 (blue and orange). These ratios are in the range for which the turbulent theory from [10] predicts an outward impurity convection.

\subsection{Ar Transport Coefficients}

Using the profiles in figure 9, the transport coefficients of the three discharges discharges have been evaluated through the G-F relation explained in sections 2 and 3. The results are shown in black in figure 10, labelled $G-F$. The plots range only up to $\rho \sim 0.65$ because of the drop in sensitivity of the SXR diodes outside of this radius. The error bars result from the linear fit of the $G-F$ relation in equation (3) and therefore do not account for the uncertainties in the electron temperature and density profiles. Moreover, the errors of the $v / D$ profiles are a simple Gaussian propagation of those of $D$ and $v$ and may thus be overestimated. The difference in amplitude of the errors between the discharges depends on the nature of the SXR data and on how much the Ar contributes to the sawtooth crashes. The case with off-axis ECRH (\#24916) exhibits "standard" sawtooth cycles with quiescent inter-crash phases and low argon contribution to the crashes themselves. This enabled the fitting of the raw SXR data after the background sawtooth subtraction (shown in figure $6 \mathrm{~b}$ ). The consequently smooth gradient-flux linear relations (figure 7) translate into small error bars in the transport coefficients. The larger error bars for discharges \#24648 and \#24709 are due to larger contributions of argon to the sawtooth crashes which do not allow the use of the fitting procedure. For the extreme case of \#24709 (background sawtooth subtraction shown in figure 6a), such large errors are due to the large oscillations in the SXR signals, a direct consequence of the strong MHD activity.

Taking the off-axis heated discharge (figure 10a) as reference, a clear increase of diffusivity and a transition of the convection velocity from negative to positive is observed when the ECRH is positioned further inward. For the most centrally heated case $(10 \mathrm{c})$, the central $D$ reaches values up to one order of magnitude higher than the reference case, while the mixed heating case (10b) exhibits an increase of $D$ up to a factor 6 at $\rho \sim 0.45$, just outside the ECRH deposition radius. The maximum convection velocity is also found around the ECRH deposition radius: $v \sim 1.0 \mathrm{~m} / \mathrm{s}$ for \#24709 and $0.5 \mathrm{~m} / \mathrm{s}$ for \#24648. For the reference discharge, $v \in[-0.4,-0.5] \mathrm{m} / \mathrm{s}$ in the range $\rho \in[0.2,0.4]$. Both results for diffusion and 

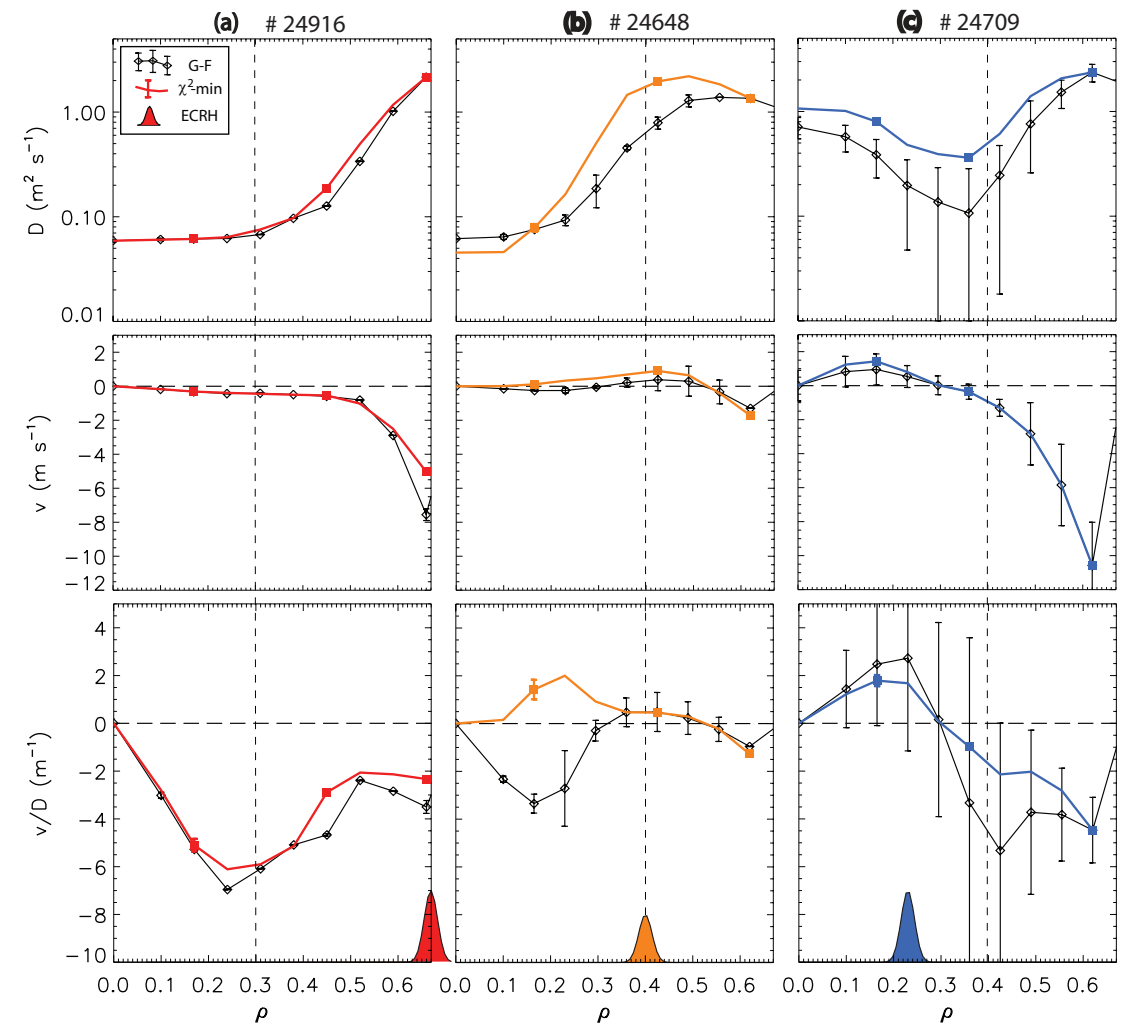

Figure 10. Argon $D$ (top), $v$ (centre) and $v / D$ (bottom) profiles for the analysed discharges (labelled on top). In black the results from the new method $(\boldsymbol{G}-\boldsymbol{F})$, in colour those from $\chi^{2}$-minimization $\left(\chi^{2}-\min\right.$, scanned knots shown as full squares). Vertical dashed lines indicate the $q=1$ surface position. Colours as in figure 9 .

convection partially confirm previous experimental findings obtained using different analysis methods $[3,4,5,6,7,8,9]$. What is striking and novel of these results is the strong localization of these effects around the ECRH deposition position. While the local increase of the convection velocity was clearly shown in [3] whereas others have seen a more global effect on the convection velocity profile $[4,5,6,7,8]$, the diffusion coefficients in all of these cases does not exhibit such localized increase around the position of deposition of ECRH (or ICRH). The results presented in this paper therefore do not contradict the old ones, but can resolve structures previously indistinguishable because of the higher spatial resolution.

\subsection{Benchmarking against $\chi^{2}$-minimization methods}

The quality of these transport coefficients has been benchmarked against $\chi^{2}$ minimization methods by scanning $D$ and $v$ and searching for the "best" set of profiles. In order to maintain the profile continuous, the scans have been performed varying three knots in the profile ( $\rho=0,0.4$ and 0.65 for the case in figure 11) and the neighbouring points have been scaled with them (light blue dashed lines). Outside of the SXR sensitivity range $(\rho>0.65$, highlighted in red in figure 11$)$ the convection velocity has been set to zero and the diffusion coefficient smoothly brought to a 


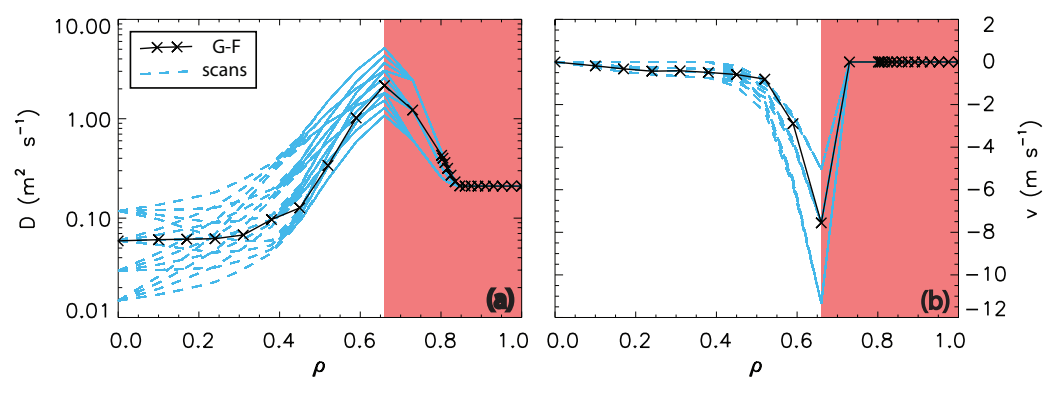

Figure 11. Example of a scan in $D$ (a) and $v$ (b) for discharge \#24916 for the application the $\chi^{2}$-minimization routine. The blind region for the SXR diagnostic is highlighted in red.

constant value ( $\sim 0.2$ in figure) so to match as best as possible the influx as seen by the SXR channel at the edge of the range. These values are therefore "mean" transport coefficients which guarantee the correct reproduction of the influx seen within the diagnostic sensitivity limit with the assumed SOL transport coefficients and recycling parameters.

For each combination of $D$ and $v$ profiles, STRAHL simulations have been performed and the simulated LoS-integrated SXR emissivity fitted to the experimental data (assumed to have a $5 \%$ error). Sawtooth crashes have been modelled as a complete flattening of the total impurity density within the mixing radius $r_{m i x}=$ $r_{i n v} \cdot \sqrt{2}$, where $r_{i n v}$ is the position of the $q=1$ surface $[28,29]$. The quality of the fit is defined by the total $\chi^{2}$ considering lines of sight of camera $I$ within the diagnostic sensitivity range. Since the LoS below and above the magnetic axis have different acquisition and amplification circuits, the comparison has been limited to the top LoS (apart from the most central LoS), thus avoiding possible systematic errors due to hardware differences. The pair of $D$ and $v$ profiles giving the lowest total $\chi^{2}$ is then used for the next scan. The final results are plotted in colour in figure 10, the scanned knots shown as coloured squares (at $\rho \sim 0.15,0.4,0.65$ ), the error bars evaluated from the percentage variation in $\chi^{2}$ with respect to the change in transport parameters [30] (setting $a=D$ or $a=v$ ):

$$
\sigma_{a}^{2}=\frac{2}{\partial^{2} \chi^{2} / \partial a^{2}}
$$

These values were exceptionally small, often smaller than the full coloured squares representing the scanned knots. The reason for this can be imputed to the $5 \%$ error assumed for the SXR raw data which may lead to a sufficiently large change in $\chi^{2}$ for small changes in either diffusion or convection. This can be easily noted in figure 12 , where the simulations using the final transport coefficients from the $\chi^{2}$-minimization are compared with the experimental data. Background profiles before the impurity puff and the background sawtooth cycles have been added to the simulated data so to compare the simulation directly with the raw signals. Since only the relative values of raw data are reliable, the simulated LoS integrals have been normalized to the maximum values of the profiles at equilibrium (end of the time range in figure 12). The agreement for all three discharges is exceptionally good, apart from a slight deviation in the sawtooth cycle and in the rise of the most edge channel of discharge \# 24709 (12c). Since this discharge exhibited unstable electron profiles inbetween sawtooth crashes (visible in the standard deviation of the profiles in figure 
9 ), simulations with time dependent $n_{e}$ and $T_{e}$ profiles has been attempted, but lead to no appreciable improvement. Plots $12 \mathrm{~d}-12 \mathrm{e}$ show the normalized SXR profiles at equilibrium, raw data with error bars in black, simulation with transport coefficients from $\chi^{2}$-minimization in red and simulation with transport coefficients from the G-F relation in dashed-blue. The profiles match very well for both sets, the G-F showing a slightly more peaked central profile for \#24648 (e) and a slightly more hollow one for \#24709 (f).

Regarding the transport coefficients, those from $\chi^{2}$-minimization (in colour in figure 10) reproduce almost exactly those from the $G-F$ relation for the off-axis

(a) \# 24916
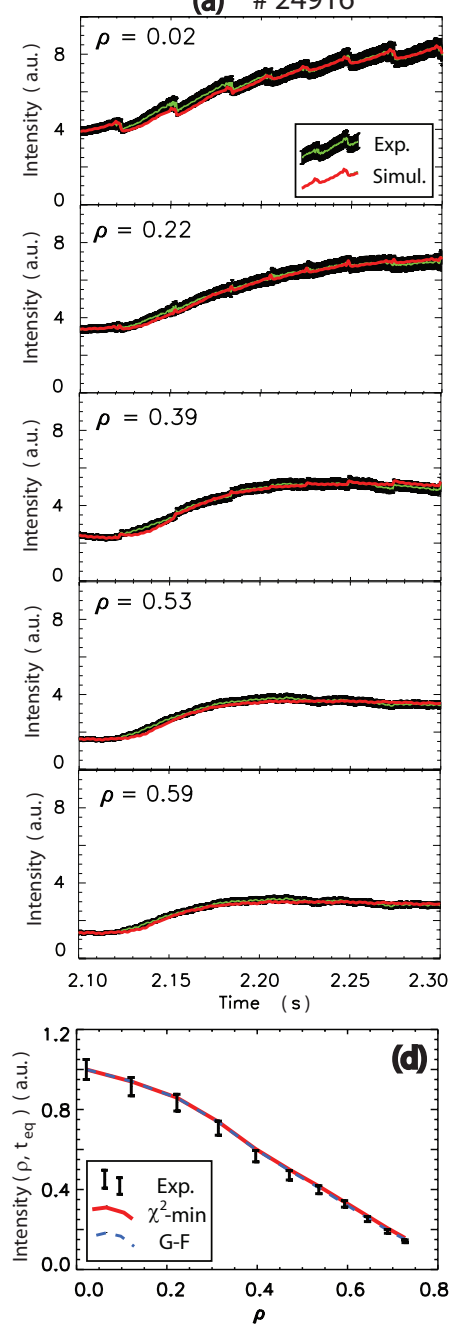

(b) \# 24648
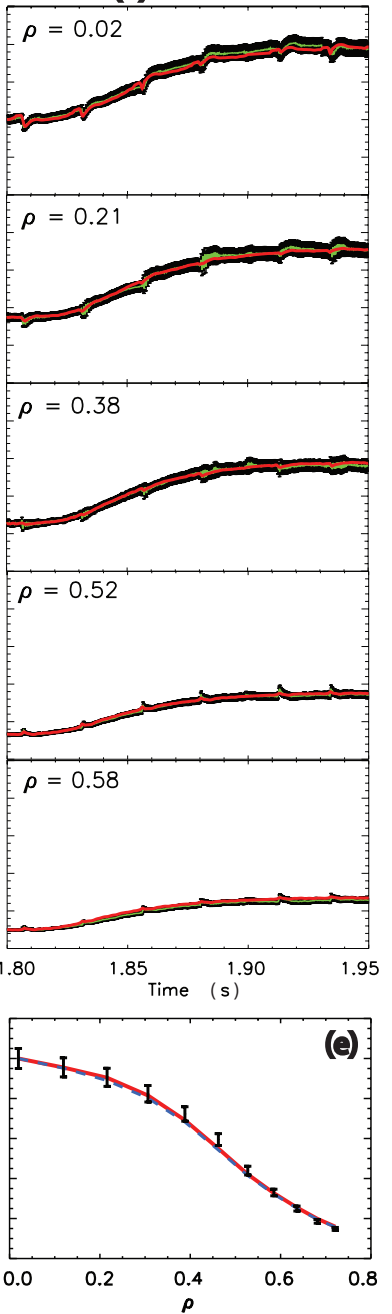

(c) \# 24709
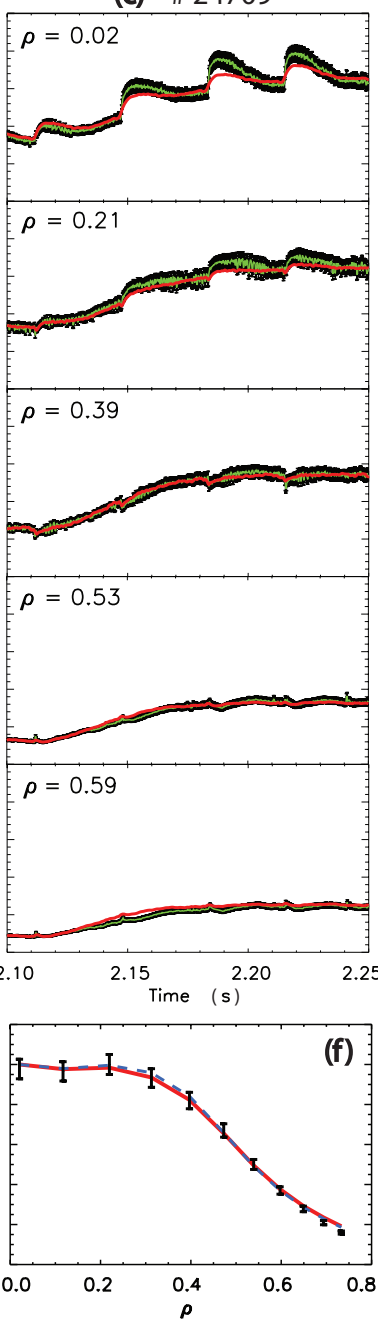

Figure 12. Comparison of raw SXR signals with the simulations performed with the transport coefficients in figure 10. Plots a-c, time evolution after the $\mathrm{Ar}$ puff: experimental signal (green), $5 \%$ error bars (black), simulations with the $\chi^{2}$ minimization transport coefficients (red). Minimum visible $\rho$ of the LoS labelled on each plot. Plots d-f, normalized equilibrium SXR profiles: experimental signal and simulations using transport coefficients from the two methods (see legend). 
ECRH discharge \#24916 (10a). The mixed heating discharge \#24648 (10b) shows instead strong deviations in the $v / D$ profile within the $q=1$ surface $(\rho \sim 0.4)$, while reproducing it perfectly outside of this radius. For discharge \#24709 (10c), the shape of the profiles is maintained, but the gradients of the diffusion coefficient are strongly reduced. In general, both the enhancement of the diffusion coefficient and the transition from negative to positive of the convection velocity are confirmed.

The complete agreement between the two methods for discharge \#24916 and the partial inconsistency inside the $q=1$ surface for discharge \#24648 could be due to the modelling of the sawtooth crash. The transport coefficients evaluated through the $G-F$ relation describe the inter-crash transport and are thus intrinsically independent of crash modelling. Without resorting to simulations it is on the other hand impossible to rigorously check the results with any measured experimental data. In contrast, $\chi^{2}$ minimization methods evaluate the transport coefficients through a direct comparison of a simulation to the measured spectroscopic data, but the result will be intrinsically dependent on the sawtooth crash model used. The one used for this work has its foundations on the theory from Kadomtsev [31] and on observations performed on "standard" sawtooth crashes [28]. Also based on Kadomtsev's model is [25], whose predicted change in sawtooth behaviour with on ECRH deposition position is in good agreement with the results shown here. On the other hand, this work assumes flat $n_{e}$ and $Z_{\text {eff }}$ profiles within the mixing radius and therefore imposes a priori the behaviour of impurity densities.

As the data presented in this paper suggests, the simple model of full flattening of the impurity density within $r_{m i x}$ describes well the "standard" situation. The discrepancies found for special cases such as those observed in discharges \#24648 and \#24709 show that this assumption is not valid, and that a different redistribution of impurities or different transport phases during the recovery from the crash may take place. In this same direction are other observations performed in different experiments which are in clear contradiction to Kadomtsev's model and which have led to the development for alternative theories to describe the crash dynamics (see [32] and references therein).

Since most impurity transport analysis rely on a model of the sawtooth crash (e.g. in [3] and in this paper through the full flattening of impurity density profile or through a strong increase in diffusion coefficient in the central region [5]), discrepancies such as those shown here were previously not detected because of the absence of an independent method. It is therefore reasonable to assert that the complete agreement between results from the $G-F$ relation and the $\chi^{2}$-minimization over the full profile for discharge \#24916 and for radii greater than the inversion radius for \#24648 provide a good first benchmarking of the new method for the evaluation of the total impurity ion density proposed in this paper. Errors in the $T_{e}$ and $n_{e}$ profiles will affect both methods in the same way and thus do not invalidate this benchmarking.

In order to better visualize the effect of the $D$ and $v$ profiles from the $G-F$ relation on the argon transport, the discharges have been simulated with STRAHL imposing the same Ar puff strength, SOL transport and recycling parameters, and excluding the sawtooth crash contributions. The time evolution of the total argon density profile is shown in figure 13, Ar density increasing from dark blue to red (colour scale of plots $13 \mathrm{~b}$ and $13 \mathrm{c}$ have been slightly expanded for visualization purposes). Despite injecting the same amount of Ar atoms, depositing the ECRH off-axis (13a) leads to core impurity density up to 6 times higher with respect to the other two cases (values labelled on the contour lines). The effect persists when the sawtooth 
crashes are added to the simulation. Moreover, the low central diffusivity in discharge \#24916 leads to a slow influx, so that equilibrium is reached only after $\sim 0.3 \mathrm{~s}$ from the Ar injection. The negative convection throughout the full profile results in a centrally peaked equilibrium impurity density. The increase in diffusivity caused by a more central ECRH deposition leads to a faster influx: the equilibrium time is reached at $\sim 0.25 s$ and $\sim 0.2 s$ after the puff for discharges \#24648 and \#24709, respectively. The positive convection translates in a profile with a drop in impurity density between $\rho \sim 0.2$ and 0.4 for \#24648 and in a hollow density profile within $\rho \sim 0.3$ for discharge \#24709. These characteristics can be also seen in the equilibrium density profile evaluated from the SXR diagnostic $n_{A r}^{S X R}\left(\rho, t_{e q}\right)$ (bottom plots in figure 13). These profiles have been averaged over $2 \mathrm{~ms}(10 \mathrm{~ms}$ for discharge \#24709) so to filter out oscillations due to noise and fast-MHD, the error bars evaluated from the deconvolution routine.
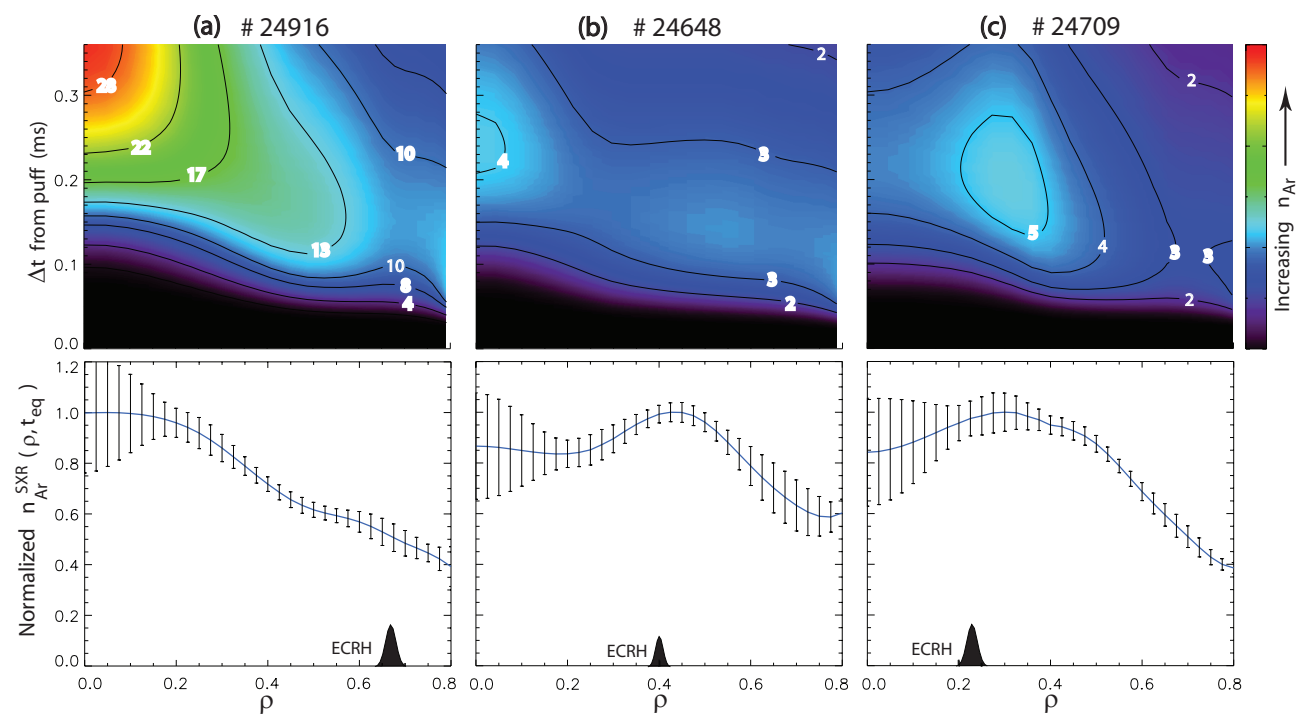

Figure 13. Top: STRAHL simulations of the $n_{A r}(\rho, t)$ after the argon injection for the analysed discharges (labelled on top) using the $G$ - $F$ transport coefficients and neglecting the sawtooth crashes. Contour values given in $10^{16} \mathrm{~m}^{-3}$. Bottom: equilibrium $n_{A r}^{S X}(r, t)$ from the deconvoluted SXR signals.

\subsection{Comparison with Theoretical Estimates}

Theoretical estimates of the neoclassical transport have been evaluated with NEOART $[33,34]$ accounting for concentrations of tungsten $(0.005 \%, 0.01 \%$ and $0.006 \%$ for discharges \# 24916, 24648 and 24709 respectively), argon (0.015\%, 0.04\% and 0.07\% respectively), carbon (1.5\%) and oxygen (0.5\%). $W$ and $A r$ values were deduced from measurements of the grazing incidence and from those of the C-Johann spectrometers respectively. Typical concentration values observed in ASDEX Upgrade have been considered for $C$ and $O$. The results (dashed lines, figures 14a and 14b) show how the central diffusivities of both off-axis and mixed heating cases are approximately neoclassical (red and orange). For completeness, a NEOART calculation has also been performed using Ar as the only impurity species, resulting in a factor $\sim 2-4$ 
underestimation of the diffusion coefficient. It is thus of major importance to consider all (most of) the impurities present in the plasma for such calculations.

The turbulent transport contributions has been evaluated using the quasi-linear calculations with the code GS2 [35, 36]. The simulations have been performed only for radii greater than the sawtooth inversion radius so to be sure of the presence of nested flux-surface geometry as assumed by the theory applied [10]. The calculations have been performed over a spectrum of 10 binormal wave numbers from $k_{y} \rho_{i}=0.15$ to $k_{y} \rho_{i}=1.0$, with logarithmic spacing. The amplitude of the fluctuating electrostatic potential $\left|\phi_{k}\right|^{2}$ has been taken proportional to $\gamma_{k} /<k_{\perp}^{2}>$, where $\gamma_{k}$ is the maximum linear growth rate and $\left\langle k_{\perp}^{2}\right\rangle$ is the perpendicular wave number averaged over the eigenfunction. For discharges \#24916 and \#24709, the results for $v / D$ at $\rho=0.615$ are $\sim-1.27 \mathrm{~m}^{-1}$ and $\sim-2.85 \mathrm{~m}^{-1}$ respectively (large red and blue circles in figure 14c). The GS2 runs underestimate the experimental absolute values by a factor $\sim 2$, but approximately reproduce the decrease in drift parameter $v / D$ observed when the ECRH is not deposited in this region (blue curve). For discharge \#24648, the calculations have been performed at three points along the profile $(\rho=0.45,0.55$ and 0.65 , orange circles in plot), qualitatively reproducing the decreasing trend of $v / D$ with increasing radius. Since the positive drift parameter is predicted to be approximately constant with increasing $Z[10]$, the $v / D$ of tungsten may be expected to be comparable to the one of argon shown here.

The positive convection observed in discharge \#24709 inside $\rho \sim 0.3$ is instead most probably not of the turbulent nature explained in [10] since, due to the strong MHD activity within the $q=1$ surface, the parallel electron motion will be highly disturbed. The transport mechanisms leading to the strong increase in $D$ and to $v>0$ might be also connected to the strong MHD activity present in-between sawtooth crashes, but clarifications in this regard are needed.

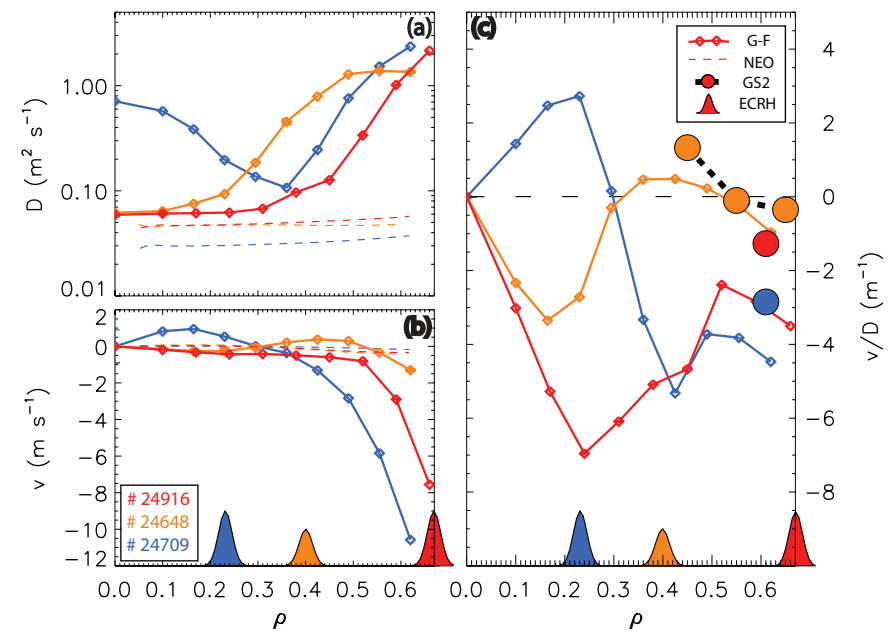

Figure 14. Profiles of $D$ (a), $v$ (b) and $v / D$ (c) of Ar for three analysed discharges. Dashed lines in (a) and (b) are the neoclassical values calculated with NEOART, large dots in (c) the results from the GS2 simulations. 


\section{Conclusions}

A novel methodology for the experimental determination of the total impurity ion density through the integrated use of a SXR diode diagnostic, a calibrated X-ray spectrometer (CSXR or C-Johann) and the 1D simulation code STRAHL has been developed. This has enabled the evaluation of the impurity transport coefficients through the linear Gradient-Flux relation of the total impurity density. The method is fast, exhibits high time and spatial resolution and is independent of sawtooth crash modelling. By modelling the this as a complete flattening within the mixing radius, first benchmarking has been successfully performed against more typical $\chi^{2}$ minimization methods. Results are in perfect agreement with those obtained using the new method for "standard" sawtooth crashes while discrepancies within the inversion radius are found for more exotic crash types. This is an indication that the sawtooth crash model may be suited only for "standard" cycles. The new method therefore exhibits positive aspects in comparison to typical $\chi^{2}$-minimization methods.

Despite these differences, both methods show an enhancement of the diffusion coefficients and the rise of a positive convection around the ECRH deposition radius for sufficiently central ECRH deposition. This leads to flat or hollow central impurity density profiles and to a global decrease in impurity density for the same amount of impurity atoms injection. The evaluated transport coefficients confirm results from previous studies using independent methods $[2,3,4,5,6,7,8,9]$ and, additionally, show that the diffusivity enhancement and the rise of an outward convection are strongly localized around the ECRH deposition radius. While the local increase of the convection velocity was clearly shown in [3] whereas others have seen a more global effect on the convection velocity profile, the diffusion coefficients in all of these cases does not exhibit such localized increase around the position of deposition of ECRH (or ICRH). To our knowledge, such localized effects are shown here for the first time.

For off-axis heating, the central diffusivity is found to be at neoclassical levels, evaluated through the use of the NEOART code considering $W, C$ and $O$ concentrations as well as $A r$. Quasi-linear gyrokinetic simulations have also been performed to test the recent turbulent theories [10]. For the most relevant discharge presented (\#24648) the theoretical $v / D$ values qualitatively reproduce the positive convection and its decreasing trend for radii outside the ECRH deposition radius. Since these effects are predicted to persist with increasing impurity charge, the effect on the tungsten density profile may be expected to be of the same order of the one which is observed here for argon. For the most central ECR-heated discharge analysed, the resulting positive convection is probably not of turbulent nature but could be caused by the strong MHD activity present within the $q=1$ surface. Since other experiments are needed to better address this problem, no further speculations are tempted in this regard.

\section{References}

[1] Fussmann G et al 1991 Plasma Phys. Controlled Fusion 331677

[2] Neu R et al 2002 Plasma Phys. Controlled Fusion 44 811-826

[3] Dux R et al 2003 Plasma Phys. Controlled Fusion 45 1815-1825

[4] Scavino E et al 2003 Plasma Physics and Controlled Fusion 45 1961-1974

[5] Puiatti M E et al 2003 Plasma Physics and Controlled Fusion 45 2011-2024

[6] Puiatti M E et al 2006 Phys. Plasmas 13042501

[7] Carraro L et al Impurity profile control in JET plasmas with radio-frequency power injection in 
Europhysics Conference Abstracts (CD-ROM, Proc. of the 34th EPS Conference on Plasma Physics, Warsaw, 2007), edited by Gasior P et al, volume 31F, pages O-4.028, Geneva, 2007, EPS

[8] Leigheb M et al 2007 Plasma Physics and Controlled Fusion 491897

[9] Giroud C et al Study of Z-dependence of impurity transport at JET in Europhysics Conference Abstracts (CD-ROM, Proc. of the 34th EPS Conference on Plasma Physics, Warsaw, 2007), edited by Gasior P et al, volume $31 \mathrm{~F}$, pages P-2.049, Geneva, 2007, EPS

[10] Angioni C et al 2006 Phys. Rev. Lett. 96 095003-1

[11] Dux R STRAHL User Manual Laborbericht 10/30, IPP Garching, September 2006

[12] Angioni C et al 2007 Plasma Phys. Controlled Fusion 49 2027-2043

[13] Parisot T et al 2008 Plasma Phys. Controlled Fusion 50055010

[14] Wade M R et al 1995 Phys. Plasmas 2 2357-2365

[15] Guirlet R et al 2010 Nucl. Fusion 50095009

[16] Igochine V Hotlink bases Soft X-ray Diagnostic on ASDEX Upgrade Technical Report 1/338, IPP, Garching, Germany, May 2010

[17] Behringer K Description of the Impurity Transport Code STRAHL Technical Report JETR(87)08, JET Joint Undertaking, Abingdon, 1987

[18] Sertoli M Local Effects of ECRH on Argon Transport at ASDEX Upgrade PhD thesis, LudwigMaximilians-Universität München, 2010

[19] Radivojevic I et al High Resolution Compact X-ray Spectrometer with Large Spherical Crystals for Ion Temperature Measurements in Europhysics Conference Abstracts (CD-ROM, Proc. of the 33rd EPS Conference on Plasma Physics, Roma, 2006), edited by De Marco F et al, volume 30I, pages P-2.148, Geneva, 2006, EPS

[20] Herrmann W et al 1998 Review of Scientific Instruments 69 3165-3175

[21] http://www.adas.ac.uk/

[22] Kallenbach A et al 2003 Nuclear Fusion 43 573-578

[23] Dux R et al 1999 Nuclear Fusion 39 1509-1522

[24] Poli E et al 2001 Computer Phys. Comm. 136 90-104

[25] Furno I et al 2001 Nucl. Fusion 41403

[26] Fischer R et al 2008 Plasma Physics and Controlled Fusion 50085009

[27] Fischer R et al Multiple Diagnostic Data Analysis of Density and Temperature Profiles in ASDEX Upgrade in Europhysics Conference Abstracts (CD-ROM, Proc. of the 36th EPS Conference on Plasma Physics, Sofia, 2009), edited by Mateev M et al, volume 33E, pages P-1.159, Geneva, 2009, EPS

[28] Seguin H, F. et al 1983 Phys. Rev. Lett. 51455

[29] Ida K et al Jan 1987 Phys. Rev. Lett. 58 116-119

[30] Bevington P Data Reduction and Error Analysis for the Physical Sciences McGraw-Hill Book Company, New York, 1969

31] Kadomtsev B B 1975 Fiz. Plazmy 1710

[32] Igochine $\mathrm{V}$ et al to be submitted to Phys. Plasmas

[33] Peeters A G 2000 Phys. Plasmas 7 268-275

[34] Dux R et al 2000 Nuclear Fusion 40 1721-1729

[35] Kotschenreuther M et al 1995 Computer Phys. Comm. $88128-140$

[36] Dorland W et al Dec 2000 Phys. Rev. Lett. 85 5579-5582 